\title{
Behavioural assessment of three chicken genotypes under free-range, semi-intensive, and intensive housing systems
}

\author{
Sohail AHMAD ${ }^{1, a, ~} \bowtie$, Athar MAHMUD ${ }^{1, b}$, Jibran HUSSAIN ${ }^{1, c}$, Khalid JAVED $^{2, d}$, \\ Muhammad USMAN ${ }^{1, \mathrm{e}}$, Muhammad WAQAS ${ }^{1, \mathrm{f}}$, Muhammad ZAID ${ }^{1, \mathrm{~g}}$
}

\begin{abstract}
${ }^{1}$ University of Veterinary and Animal Sciences, Faculty of Animal Production and Technology, Department of Poultry Production, Lahore, Pakistan; ${ }^{2}$ University of Veterinary and Animal Sciences, Lahore, Pakistan, Faculty of Animal Production and Technology, Department of Livestock Production, Lahore, Pakistan. aORCID: 0000-0002-0390-3083; b ORCID: 0000-0002-2106-4113; ' ORCID: 0000-0002-4837-375X; dORCID: 0000-0001-7610-9967; ${ }^{\mathrm{e} O R C I D: 0000-0002-3128-3969 ;}$ f ORCID: 0000-0002-9972-8426; gORCID: 0000-0002-1688-4504.
\end{abstract}

\begin{abstract}
The present study evaluated the effects of housing systems (free-range, semi-intensive, and intensive) on the behaviour of chickens over 10 weeks period (7-16 weeks of age. A total of 360 birds were selected and subjected to different housing systems. A Randomized Complete Block Design (RCBD) considered the following: 3 genotypes (RNN, BNN, and NN) $\times 2$ sexes $(30$ cockerels and 30 pullets $=60 /$ genotype $) \times 3$ housing systems (free-range, semi-intensive, and intensive) $=18$ experimental units with 20 birds per unit $=360$ birds. Regarding behavioural response, male birds under the intensive system were more aggressive and showed more sitting and standing behaviour followed by semi-intensive and free-range systems. Jumping, running, walking and wing flapping behaviours were higher in semi-intensive birds followed by free-range and intensive systems. Regarding females, RNN and BNN chicken revealed higher running behaviour than NN. In terms of housing systems, birds reared in the intensive system were more aggressive and showed an increased frequency of sitting and standing behaviours followed by semi-intensive and free-range systems. Birds under the free-range system spent most of their time in feeding and wing flapping followed by semi-intensive and intensive housing systems. Jumping, running, and walking was more pronounced in the semi-intensive system followed by a free-range and intensive system. It was concluded that RNN and BNN chickens expressed more natural behaviours under semi-intensive and freerange systems than NN chickens; hence, crossbred chickens could be reared under such types of environments to achieve their maximum genetic potential.
\end{abstract}

Keywords: Behavioural response, chicken, crossbred, housing system, purebred.

\section{Introduction}

In developing countries, the popularity of backyard type chicken meat is increasing day by day reason behind its unique flavour and texture which is regarded as a greater delicacy than commercial lines. The local market for indigenous chicken has increased substantially, which stimulated its export potential. Therefore, changing the housing system of these chickens from an extensive backyard to a semi-intensive or intensive system could increase the income for small scale rural poultry farmers $(1,8)$. In Pakistan, there are three indigenous breeds viz., Desi (non-descript), Naked Neck, and Aseel. Among these, Naked Neck has greater production potential as the other two breeds are very late maturing, slower growing, and produce fewer and lighter eggs. The Naked Neck has the potential to be developed as a dual-purpose breed and kept by farmers for both egg and meat production due to its better survivability in adverse rural conditions. The Naked Neck gene is also known to have the potential to alleviate heat stress in birds (19). Rhode Island Red is originally bred in Adamsville (Rhode Island) and is an American chicken breed popular for its better meat and egg production. The black-breasted red Malay cock was considered as one of the foundation sires of this breed and officially displayed at Smithsonian Institution as the father of Rhode Island Red (1). Black Australorp is a highly successful commercial line, originating from Australia via selective breeding with Black Orpington $(1,2)$. It is a dual- 
purpose breed having dark textured meat and is a good producer of brown eggs (23). This breed holds a unique record of producing 364 eggs in 365 days (5). Crossbreeding is a useful technique to exploit genetic variation and is generally termed as the mating of two individuals having different genetic makeup (21). Crossbreeding increases heterozygosity in the population (15), with the main objective being to produce offspring that has desirable qualities of both parent lineages (18). Crossbreeding is generally helpful not only as it creates combinations of desirable characters, but is also produces heterosis or hybrid vigour. It is also responsible for rapid change in population with the introduction of new breeds (13).

Behaviour is an overt expression of what's going on internally, inside the bird, or as a result of external circumstances. It is a reaction to environmental changes that largely depends upon both genetic and non-genetic factors. Consequently, a better understanding of environmental influence could be helpful for indemnification and quantification of welfare. Environment and housing conditions are generally considered as important factors that directly influence chicken behaviour. Therefore, environmental enrichment may influence a bird's behaviour and provide a large number of behavioural opportunities. Chicken behaviour depends on the housing environment (6).

The free-range system is generally considered as effective from the bird's welfare point of view because it allows the bird to perform and exhibit natural behaviours and provide additional space for exercise. A free-range system results in more favourable meat quality traits especially chemical composition although this system has some demerits, such as exposure to pathogens and adverse weather conditions (7). Farmers might avoid this system for several reasons that include lack of appropriate shelter, weight reduction of young birds being outside. Furthermore, the free-range system is associated with a reduction in weight gain and poor feed conversion efficiency in meat-type birds (3).

The housing system may influence the healthpromoting activities of birds which ultimately affects the properties of meat and eggs but the significance of genotype should not be undervalued when selecting birds for any housing environment. Backyard chicken genotypes perform substantially better in the free-range system; however, a better understanding of how different housing systems can influence the behaviour of different genotypes is still required. Therefore, the objective of this study was to investigate the behavioural response of three chicken genotypes under free-range, semi-intensive, and intensive housing systems. We hypothesized that different chicken genotypes behave differently in alternative housing environments.

\section{Materials and Methods}

This study was conducted at the Department of Poultry Production, the University of Veterinary and Animal Sciences, Ravi Campus, Pattoki, Pakistan. This city experiences a normally hot and humid tropical climate with temperatures ranging from $13{ }^{\circ} \mathrm{C}$ in winter and +45 ${ }^{\circ} \mathrm{C}$ in summer.

Ethics: The care and use of birds were by the laws and regulations of Pakistan and was approved by the Committee of Ethical Handling of Experimental Birds (No. DR/124), the University of Veterinary and Animal Sciences (UVAS), Lahore, Pakistan.

Population Size: A baseline population of pure Naked Neck (NN), Rhode Island Red (RIR), and Black Australorp (BAL) already maintained at Indigenous Chicken Genetic Resource Centre (ICGRC), UVAS, Pakistan comprising 450 birds ( 90 male +360 female), 150 from each breed (30 males +120 females). RIR and BAL males were crossed with NN females and their progeny were selected for this experimentation termed as crossbreds. Besides, NN males are also crossed with NN females and their progeny were selected for this experimentation and named as purebred. A total of 480, day-old chicks comprising 160 from each genotype (RIR $\times \mathrm{NN}=\mathrm{RNN}, \mathrm{BAL} \times \mathrm{NN}=\mathrm{BNN}$, and $\mathrm{NN} \times \mathrm{NN}=\mathrm{NN}$ ) were brooded at a well ventilated open-sided house with standard managemental conditions till 6th weeks of life. Birds were provided by ration formulated according to the recommendation of Leeson and Summers (10), and daily bird's allowance was increasing as per requirement. In the brooding period, birds were vaccinated against Newcastle Disease and Infectious Bronchitis according to the schedule of the local area. After 6 weeks, when birds were acclimatized to the environment experimentation was started and lasted 10 weeks.

During the growing phase (7-16 weeks), 360 birds were selected and subjected to different housing systems. A Randomized Complete Block Design (RCBD) considered the following: 3 genotypes (RNN, BNN, and $\mathrm{NN}) \times 2$ sexes $(30$ cockerels and 30 pullets $=60 /$ genotype) $\times 3$ housing systems (free-range, semiintensive, and intensive) $=18$ experimental units with 20 birds per unit $=360$ birds.

Free-range, semi-intensive and intensive system: All the experimental birds were maintained in an opensided shed $(6.1 \mathrm{~m} \mathrm{~L} \times 6.1 \mathrm{~m} \mathrm{~W} \times 3.66 \mathrm{~m} \mathrm{H})$ oriented east to west. Birds were reared on the floor having a bedding material of rice husk at stocking density $\left(0.19 \mathrm{~m}^{2}\right.$ per bird). Freshwater was provided through a manual drinker. A patch of fertile land measuring $(10 \mathrm{~m} \mathrm{~L} \times 2.99 \mathrm{~m} \mathrm{~W}$; stocking density $=0.23 \mathrm{~m}^{2} /$ bird) located in front of the shed was used as a range area. Seasonal leguminous and non-leguminous plants were grown in the range area. In the ranging area, two rows were made by using fishing 
nets (one for free-range and the other for semi-intensive). Fresh ad libitum water was ensured through manual drinkers (Henan Lima Machinery Co., Ltd., China). For the protection of the birds, a $2.44 \mathrm{~m}$ high wire-mesh enclosure was installed which surrounded the range area. In free-range and semi-intensive systems, birds were given access to vegetation from 06:00 AM to 06:00 PM and 06:00 AM to 12:00 PM, respectively (Figure 1, 2). The free-range group was offered with a $50 \%$ grower ration in the evening.

In an intensive housing system, birds were maintained in the battery cage system (FACCO, Poultry Equipment-C3) situated in a well-ventilated poultry shed and were fed commercial grower ration as per the recommendation of Leeson and Summers (10). The daily allowance was increased corresponding to their growth and requirement (Table 1, 2, and 3).
Table 1. Nutrient composition of experimental ration for different phases.

\begin{tabular}{lcc}
\hline Nutrients & $\begin{array}{c}\text { Starter } \\
\text { (0-6 weeks) }\end{array}$ & $\begin{array}{c}\text { Grower } \\
\text { (7-16 weeks) }\end{array}$ \\
\hline CP (\%) & 16 & 14 \\
ME (Kcal/Kg) & 2900 & 2850 \\
Ca (\%) & 0.90 & 0.87 \\
Av. P (\%) & 0.40 & 0.38 \\
Lysine (\%) & 0.80 & 0.70 \\
Methionine (\%) & 0.33 & 0.30 \\
Na (\%) & 0.19 & 0.19 \\
\hline
\end{tabular}

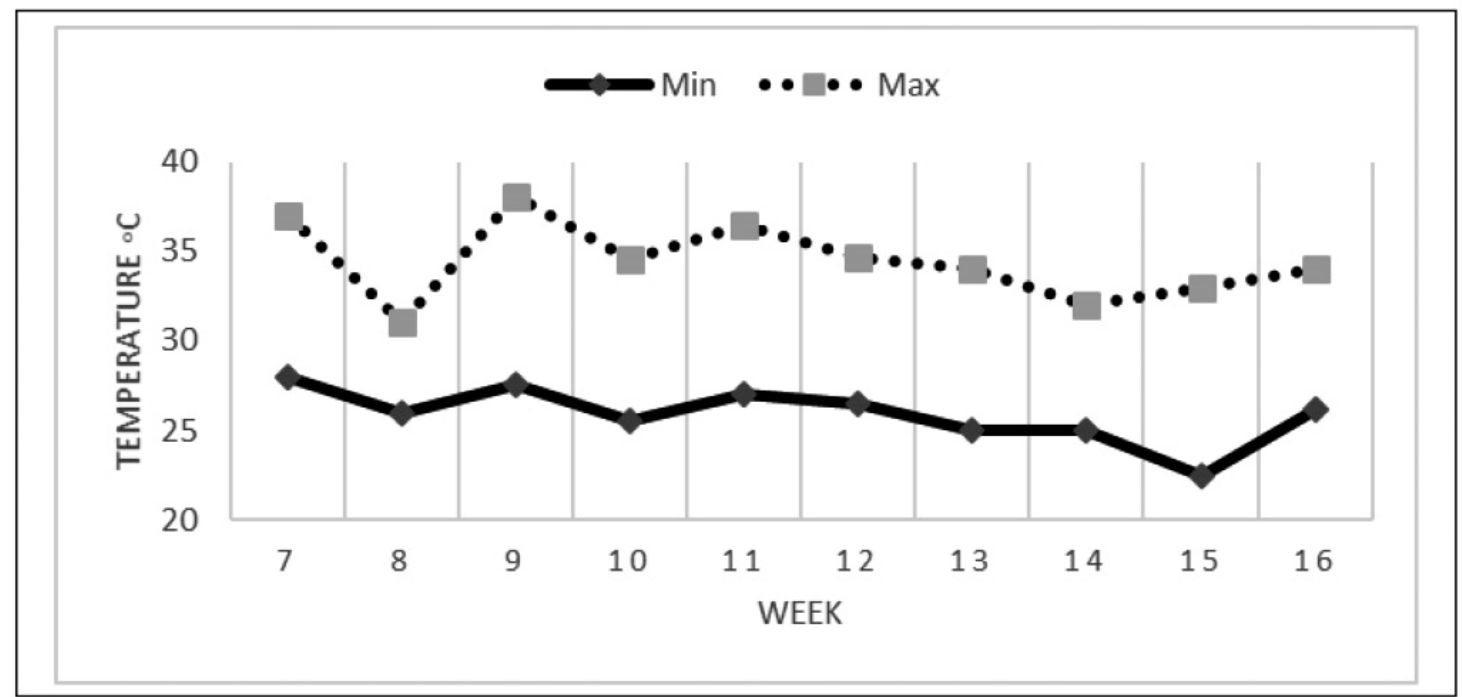

Figure 1. Trend of weekly temperature during experimentation.

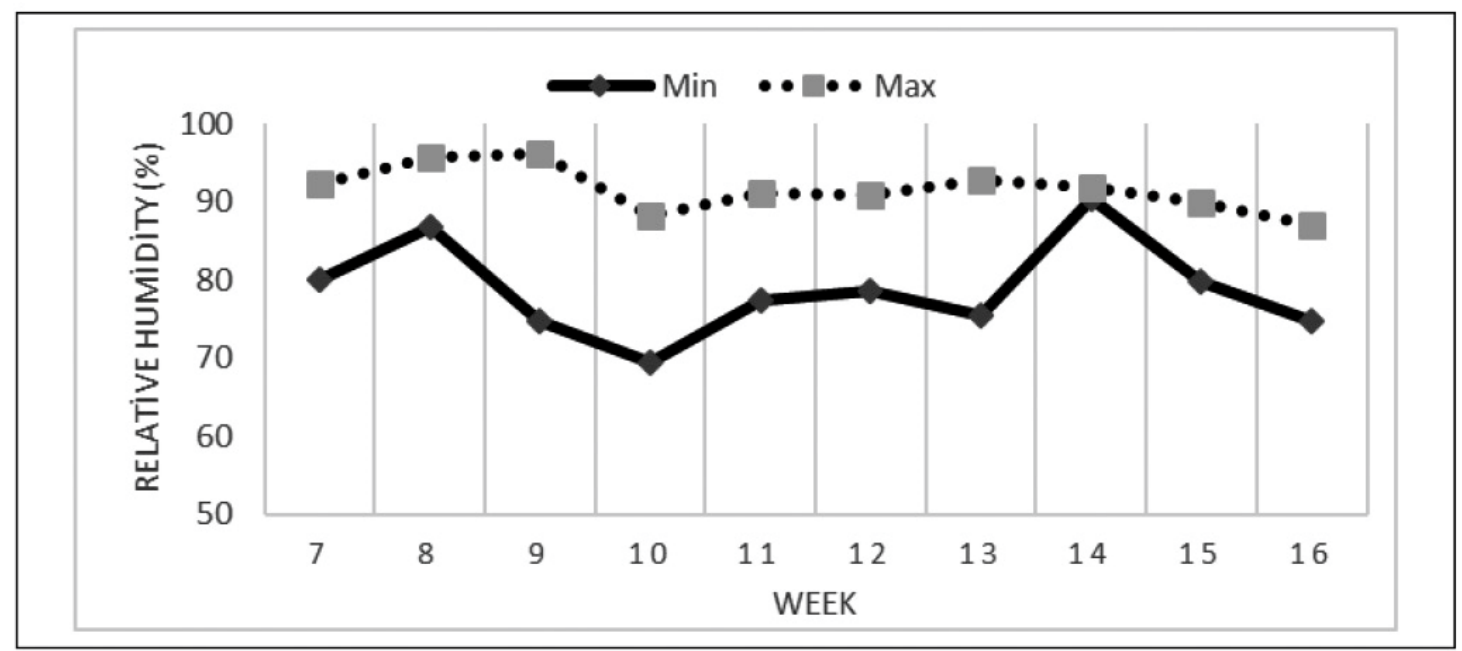

Figure 2. Trend of weekly relative humidity during experimentation. 
Table 2. Weekly feed allowance (g/bird) in the growing phase (7-16 weeks).

\begin{tabular}{cccc}
\hline & \multicolumn{3}{c}{ Housing System } \\
\cline { 2 - 4 } Age (Week) & Free Range & $\begin{array}{c}\text { Semi- } \\
\text { intensive }\end{array}$ & Intensive \\
\hline 7 & 0 & 12 & 24 \\
8 & 0 & 14 & 28 \\
9 & 0 & 15 & 30 \\
10 & 0 & 15 & 30 \\
11 & 0 & 17 & 34 \\
12 & 0 & 18 & 36 \\
13 & 0 & 19 & 38 \\
14 & 0 & 19 & 38 \\
15 & 0 & 20 & 40 \\
16 & 0 & 21 & 42 \\
\hline
\end{tabular}

Table 3. Composition of Experimental Rations (Starter and Grower).

\begin{tabular}{lcc}
\hline $\begin{array}{l}\text { Feed Ingredient } \\
(\%)\end{array}$ & $\begin{array}{c}\text { Starter } \\
\text { (0-6 weeks) }\end{array}$ & $\begin{array}{c}\text { Grower } \\
\text { (7-16 weeks) }\end{array}$ \\
\hline Corn & 60.63 & 62.55 \\
Soybean Meal & 33.34 & 32.33 \\
Fish Meal & 2.00 & 0.00 \\
Soybean Oil & 2.00 & 3.00 \\
DCP & 1.50 & 1.70 \\
NaCl & 0.30 & 0.30 \\
Methionine & 0.23 & 0.12 \\
Total & 100 & 100 \\
\hline
\end{tabular}

Parameters evaluated: Each experimental bird was wing tagged. The observation was conveyed weekly, between 11:00 AM to 01:00 PM and the time spent in each behaviour was noted (Table 4). The behaviour repertoire was recorded according to the focal animal sampling method adopted by Rehman et al. (16). Before visual scanning, at least a half-hour elapsed that allowing the birds to redistribute normally as the observers moved across the replicates. During the observer training period, birds were habituated to the presence of the observers and the beep sounds of the stopwatch. Birds were randomly selected ( 3 birds per replicate) and marked on the neck and tail feather with a white colour stain for identification. All four observers were positioned near birds, avoiding any interruption in their activities. The birds were observed for 15 minutes by using stopwatches fixed at 5 seconds beeps and the behavioural range was recorded individually (time spent in each behaviour). After each observation, the observers paused for 5 minutes, allowing the next focal bird to regain its normal position. The behaviour of chicken was included walking, jumping, running, feeding, standing, sitting, aggressiveness, dust bathing, and wing flapping, and behaviour occurrence proportions were
Table 4. Ethogram of behavioural pattern ${ }^{1}$.

\begin{tabular}{ll}
\hline Behaviour & \multicolumn{1}{c}{ Definition } \\
\hline Walking & $\begin{array}{l}\text { The bird moves at least two steps in } \\
\text { succession. This may or may not include } \\
\text { scratching with feet. }\end{array}$ \\
Jumping & $\begin{array}{l}\text { Movement of bird in a rebound by leaping } \\
\text { with all feet off the ground. }\end{array}$ \\
Running & An activity of wing-assisted running. \\
Feeding / & $\begin{array}{l}\text { The bird's head is located inside the feeder / } \\
\text { towards forage and carrying out pecking, } \\
\text { Foraging }\end{array}$ \\
manipulating, or ingesting feed once or \\
repeatedly.
\end{tabular}

calculated. Ethogram of the behavioural pattern are presented in Table $4(4,12)$.

Statistical analysis: The experiment was set up as an RCBD with the following model:

$$
\mathrm{Y}_{\mathrm{ijk}}=\mu+\beta_{\mathrm{i}}+\tau_{\mathrm{j}}+(\beta \times \tau)_{\mathrm{ij}}+\epsilon_{\mathrm{ijk}}
$$

Where,

$\mathrm{Y}_{\mathrm{ijk}}=$ Observation of dependent variable recorded on $\mathrm{j}^{\text {th }}$ Housing System in $\mathrm{i}^{\text {th }}$ Block

$\mu=$ Overall population mean

$\beta_{\mathrm{i}}=$ Effect of $i^{\text {th }}$ Block $(i=1,2,3)$

$\tau_{j}=$ Effect of $j^{\text {th }}$ Housing System $(j=1,2,3)$

$(\beta \times \tau)_{\mathrm{ij}}=$ Interaction between the block and

housing system

$\epsilon_{\mathrm{ijk}}=$ Residual error of $\mathrm{k}^{\text {th }}$ observation on $\mathrm{j}^{\text {th }}$ treatment in $i^{\text {th }}$ block NID $\sim 0, \sigma^{2}$

The effect of different chicken genotypes and housing systems on behavioural traits were evaluated through Factorial ANOVA using General Linear Model Procedures in SAS software (version, 9.1.3). For pair viz comparison of means, the Tukey Kramer test (22) was applied and differences were considered statistically significant at $\mathrm{P} \leq 0.05$. All the data were represented as least square mean \pm standard errors. 


\section{Results}

The behavioural pattern differed among genotypes and the interactions between genotypes and housing systems (Tables 5, 6, 7, and, 8). Regarding males, significant differences were observed among different housing systems in terms of aggression, dust bathing, feeding, jumping, running, sitting, standing, walking, and wing flapping. Birds under the intensive-system were more aggressive following by semi-intensive and freerange systems $(\mathrm{P}<0.001)$. Dust bathing was more pronounced in free-range birds than the semi-intensive systems $(15.15$ vs. $12.43 \%$; $\mathrm{P}<0.001)$. Birds were spending most of the time feeding in the free-range system, followed in semi-intensive and intensive systems $(\mathrm{P}<0.001)$. Jumping $(\mathrm{P}<0.001)$ and running $(\mathrm{P}<0.001)$ behaviours were more frequent in the semi-intensive system followed by free-range and intensive systems.

Table 5. Effect of genotype and housing system on male behavioural traits (7-16 weeks $\left.{ }^{1}\right)$.

\begin{tabular}{|c|c|c|c|c|c|c|c|c|}
\hline \multirow{2}{*}{ Trait $^{2}$} & \multicolumn{3}{|c|}{ Genotype } & \multirow{2}{*}{ P-value } & \multicolumn{3}{|c|}{ Housing System } & \multirow{2}{*}{ P-value } \\
\hline & RNN $(n=60)$ & BNN $(n=60)$ & NN $(n=60)$ & & FR $(n=60)$ & SI $(n=60)$ & $I(n=60)$ & \\
\hline WAK & $13.29 \pm 1.38$ & $13.21 \pm 1.38$ & $13.25 \pm 1.38$ & 0.431 & $13.17 \pm 0.05^{\mathrm{b}}$ & $26.27 \pm 0.06^{\mathrm{a}}$ & $0.31 \pm 0.00^{c}$ & $<0.001$ \\
\hline JMP & $0.74 \pm 0.07$ & $0.74 \pm 0.07$ & $0.73 \pm 0.07$ & 0.467 & $0.92 \pm 0.01^{\mathrm{b}}$ & $1.25 \pm 0.01^{\mathrm{a}}$ & $0.04 \pm 0.00^{c}$ & $<0.001$ \\
\hline RUN & $7.82 \pm 0.78$ & $7.92 \pm 0.80$ & $7.84 \pm 0.78$ & 0.199 & $8.89 \pm 0.04^{b}$ & $14.69 \pm 0.05^{\mathrm{a}}$ & $0.00 \pm 0.00^{c}$ & $<0.001$ \\
\hline FD & $18.09 \pm 0.30$ & $18.06 \pm 0.32$ & $18.10 \pm 0.31$ & 0.797 & $21.28 \pm 0.04^{\mathrm{a}}$ & $17.30 \pm 0.05^{\mathrm{b}}$ & $15.67 \pm 0.04^{\mathrm{c}}$ & $<0.001$ \\
\hline STD & $14.30 \pm 1.19$ & $14.25 \pm 1.20$ & $14.22 \pm 1.19$ & 0.286 & $9.11 \pm 0.03^{b}$ & $6.51 \pm 0.02^{\mathrm{c}}$ & $27.14 \pm 0.05^{\mathrm{a}}$ & $<0.001$ \\
\hline SIT & $13.54 \pm 1.09$ & $13.55 \pm 1.08$ & $13.58 \pm 1.09$ & 0.713 & $8.42 \pm 0.03^{b}$ & $6.92 \pm 0.02^{\mathrm{c}}$ & $25.33 \pm 0.05^{\mathrm{a}}$ & $<0.001$ \\
\hline AGR & $1.72 \pm 0.08$ & $1.71 \pm 0.08$ & $1.72 \pm 0.08$ & 0.804 & $1.12 \pm 0.01^{\mathrm{c}}$ & $1.51 \pm 0.01^{\mathrm{b}}$ & $2.52 \pm 0.02^{\mathrm{a}}$ & $<0.001$ \\
\hline DB & $13.79 \pm 0.22$ & $13.81 \pm 0.23$ & $13.79 \pm 0.22$ & 0.964 & $15.15 \pm 0.04^{\mathrm{a}}$ & $12.43 \pm 0.04^{b}$ & -- & $<0.001$ \\
\hline WF & $10.66 \pm 0.92$ & $10.66 \pm 0.92$ & $10.73 \pm 0.93$ & 0.397 & $15.08 \pm 0.04^{\mathrm{b}}$ & $16.28 \pm 0.05^{\mathrm{a}}$ & $0.69 \pm 0.00^{c}$ & $<0.001$ \\
\hline
\end{tabular}

${ }^{\mathrm{a}-\mathrm{c}}$ Means in a row with no common superscript differ significantly at $\mathrm{P} \leq 0.05$.

${ }^{1}$ Values are least square mean \pm standard error, average of each behaviour repertoire from 7-16 weeks

${ }^{2}$ Traits are presented as percentage of time spent in different behavioural activities

RNN $=$ Rhode Island Red $\times$ Naked Neck; BNN $=$ Black Australorp $\times$ Naked Neck; NN $=$ Naked Neck; FR $=$ Free Range; SI $=$ Semi Intensive; I = Intensive; WAK = Walking; JMP = Jumping; RUN = Running; FD = Feeding; STD = Standing; SIT = Sitting; AGR = Aggressiveness; DB = Dust Bathing; WF = Wing Flapping.

Table 6. Interaction effects (genotype $\times$ housing system) on male behavioural traits $\left(7-16\right.$ weeks $\left.{ }^{1}\right)$.

\begin{tabular}{|c|c|c|c|c|c|c|c|c|c|c|}
\hline \multirow[b]{2}{*}{ Trait $^{2}$} & \multicolumn{3}{|c|}{ RNN } & \multicolumn{3}{|c|}{ BNN } & \multicolumn{3}{|c|}{$\mathbf{N N}$} & \multirow[b]{2}{*}{ P-value } \\
\hline & $\begin{array}{c}\text { FR } \\
(\mathbf{n}=\mathbf{2 0})\end{array}$ & $\begin{array}{c}\text { SI } \\
(n=20)\end{array}$ & $\begin{array}{c}I \\
(n=20)\end{array}$ & $\begin{array}{c}\text { FR } \\
(\mathbf{n}=\mathbf{2 0})\end{array}$ & $\begin{array}{c}\text { SI } \\
(\mathbf{n}=\mathbf{2 0})\end{array}$ & $\begin{array}{c}I \\
(\mathbf{n}=\mathbf{2 0})\end{array}$ & $\begin{array}{c}\text { FR } \\
(n=20)\end{array}$ & $\begin{array}{c}\text { SI } \\
(n=20)\end{array}$ & $\begin{array}{c}I \\
(n=20)\end{array}$ & \\
\hline WAK & $\begin{array}{c}13.27 \\
\pm 0.06^{\mathrm{b}}\end{array}$ & $\begin{array}{c}26.29 \\
\pm 0.11^{\mathrm{a}}\end{array}$ & $\begin{array}{c}0.31 \\
\pm 0.01^{\mathrm{c}}\end{array}$ & $\begin{array}{c}13.12 \\
\pm 0.10^{\mathrm{b}}\end{array}$ & $\begin{array}{c}26.19 \\
\pm 0.10^{\mathrm{a}}\end{array}$ & $\begin{array}{c}0.31 \\
\pm 0.00^{\mathrm{c}}\end{array}$ & $\begin{array}{c}13.14 \\
\pm 0.10^{\mathrm{b}}\end{array}$ & $\begin{array}{c}26.31 \\
\pm 0.11^{\mathrm{a}}\end{array}$ & $\begin{array}{c}0.31 \\
\pm 0.01^{\mathrm{c}}\end{array}$ & $<0.001$ \\
\hline JMP & $\begin{array}{c}0.94 \\
\pm 0.02^{\mathrm{b}}\end{array}$ & $\begin{array}{c}1.25 \\
\pm 0.02^{\mathrm{a}}\end{array}$ & $\begin{array}{c}0.04 \\
\pm 0.00^{\mathrm{c}}\end{array}$ & $\begin{array}{c}0.92 \\
\pm 0.01^{\mathrm{b}}\end{array}$ & $\begin{array}{c}1.25 \\
\pm 0.02^{\mathrm{a}}\end{array}$ & $\begin{array}{c}0.04 \\
\pm 0.00^{\mathrm{c}}\end{array}$ & $\begin{array}{c}0.90 \\
\pm 0.02^{\mathrm{b}}\end{array}$ & $\begin{array}{c}1.25 \\
\pm 0.01^{\mathrm{a}}\end{array}$ & $\begin{array}{c}0.04 \\
\pm 0.00^{\mathrm{c}}\end{array}$ & $<0.001$ \\
\hline RUN & $\begin{array}{c}8.87 \\
\pm 0.05^{\mathrm{b}}\end{array}$ & $\begin{array}{c}14.60 \\
\pm 0.10^{\mathrm{a}}\end{array}$ & $\begin{array}{c}0.00 \\
\pm 0.00^{\mathrm{c}}\end{array}$ & $\begin{array}{c}8.90 \\
\pm 0.09^{\mathrm{b}}\end{array}$ & $\begin{array}{c}14.85 \\
\pm 0.09^{\mathrm{a}}\end{array}$ & $\begin{array}{c}0.00 \\
\pm 0.00^{\mathrm{c}}\end{array}$ & $\begin{array}{c}8.91 \\
\pm 0.08^{\mathrm{b}}\end{array}$ & $\begin{array}{c}14.60 \\
\pm 0.08^{\mathrm{a}}\end{array}$ & $\begin{array}{c}0.00 \\
\pm 0.00^{\mathrm{c}}\end{array}$ & $<0.001$ \\
\hline FD & $\begin{array}{c}21.22 \\
\pm 0.06^{\mathrm{a}}\end{array}$ & $\begin{array}{c}17.28 \\
\pm 0.07^{\mathrm{b}}\end{array}$ & $\begin{array}{c}15.76 \\
\pm 0.06^{c}\end{array}$ & $\begin{array}{c}21.35 \\
\pm 0.07^{\mathrm{a}}\end{array}$ & $\begin{array}{c}17.30 \\
\pm 0.08^{b}\end{array}$ & $\begin{array}{c}15.54 \\
\pm 0.09^{c}\end{array}$ & $\begin{array}{c}21.27 \\
\pm 0.08^{\mathrm{a}}\end{array}$ & $\begin{array}{c}17.32 \\
\pm 0.10^{\mathrm{b}}\end{array}$ & $\begin{array}{c}15.72 \\
\pm 0.08^{c}\end{array}$ & $<0.001$ \\
\hline STD & $\begin{array}{c}9.18 \\
\pm 0.04^{\mathrm{b}}\end{array}$ & $\begin{array}{c}6.52 \\
\pm 0.03^{\mathrm{c}}\end{array}$ & $\begin{array}{c}27.18 \\
\pm 0.07^{\mathrm{a}}\end{array}$ & $\begin{array}{c}9.09 \\
\pm 0.06^{\mathrm{b}}\end{array}$ & $\begin{array}{c}6.46 \\
\pm 0.05^{\mathrm{c}}\end{array}$ & $\begin{array}{c}27.21 \\
\pm 0.08^{\mathrm{a}}\end{array}$ & $\begin{array}{c}9.07 \\
\pm 0.06^{\mathrm{b}}\end{array}$ & $\begin{array}{c}6.56 \\
\pm 0.03^{\mathrm{c}}\end{array}$ & $\begin{array}{c}27.03 \\
\pm 0.09^{\mathrm{a}}\end{array}$ & $<0.001$ \\
\hline SIT & $\begin{array}{c}8.44 \\
\pm 0.06^{\mathrm{b}}\end{array}$ & $\begin{array}{c}6.82 \\
\pm 0.04^{c}\end{array}$ & $\begin{array}{c}25.36 \\
\pm 0.10^{\mathrm{a}}\end{array}$ & $\begin{array}{c}8.40 \\
\pm 0.07^{\mathrm{b}}\end{array}$ & $\begin{array}{c}6.97 \\
\pm 0.05^{\mathrm{c}}\end{array}$ & $\begin{array}{c}25.29 \\
\pm 0.07^{\mathrm{a}}\end{array}$ & $\begin{array}{c}8.41 \\
\pm 0.06^{\mathrm{b}}\end{array}$ & $\begin{array}{c}6.98 \\
\pm 0.03^{\mathrm{c}}\end{array}$ & $\begin{array}{c}25.36 \\
\pm 0.10^{\mathrm{a}}\end{array}$ & $<0.001$ \\
\hline AGR & $\begin{array}{c}1.11 \\
\pm 0.02^{\mathrm{c}}\end{array}$ & $\begin{array}{c}1.50 \\
\pm 0.02^{\mathrm{b}}\end{array}$ & $\begin{array}{c}2.56 \\
\pm 0.05^{\mathrm{a}}\end{array}$ & $\begin{array}{c}1.12 \\
\pm 0.02^{\mathrm{c}}\end{array}$ & $\begin{array}{c}1.51 \\
\pm 0.02^{\mathrm{b}}\end{array}$ & $\begin{array}{c}2.49 \\
\pm 0.04^{\mathrm{a}}\end{array}$ & $\begin{array}{c}1.13 \\
\pm 0.02^{\mathrm{c}}\end{array}$ & $\begin{array}{c}1.53 \\
\pm 0.02^{\mathrm{b}}\end{array}$ & $\begin{array}{c}2.50 \\
\pm 0.04^{\mathrm{a}}\end{array}$ & $<0.001$ \\
\hline DB & $\begin{array}{c}15.11 \\
\pm 0.07^{\mathrm{a}}\end{array}$ & $\begin{array}{c}12.47 \\
\pm 0.08^{b}\end{array}$ & -- & $\begin{array}{c}15.19 \\
\pm 0.08^{\mathrm{a}}\end{array}$ & $\begin{array}{c}12.42 \\
\pm 0.08^{b}\end{array}$ & -- & $\begin{array}{c}15.16 \\
\pm 0.05^{\mathrm{a}}\end{array}$ & $\begin{array}{c}12.41 \\
\pm 0.06^{b}\end{array}$ & -- & $<0.001$ \\
\hline WF & $\begin{array}{c}15.02 \\
\pm 0.07^{\mathrm{b}}\end{array}$ & $\begin{array}{c}16.26 \\
\pm 0.12^{\mathrm{a}} \\
\end{array}$ & $\begin{array}{c}0.69 \\
\pm 0.01^{\mathrm{c}}\end{array}$ & $\begin{array}{c}15.04 \\
\pm 0.06^{\mathrm{b}}\end{array}$ & $\begin{array}{c}16.26 \\
\pm 0.09^{\mathrm{a}}\end{array}$ & $\begin{array}{c}0.68 \\
\pm 0.01^{\mathrm{c}}\end{array}$ & $\begin{array}{c}15.18 \\
\pm 0.09^{\mathrm{b}}\end{array}$ & $\begin{array}{c}16.32 \\
\pm 0.08^{\mathrm{a}}\end{array}$ & $\begin{array}{c}0.69 \\
\pm 0.01^{\mathrm{c}} \\
\end{array}$ & $<0.001$ \\
\hline
\end{tabular}

${ }^{\mathrm{a}-\mathrm{c}}$ Means in a row with no common superscript differ significantly at $\mathrm{P} \leq 0.05$.

${ }^{1}$ Values are least-square mean \pm standard error, an average of each behaviour repertoire from 7-16 weeks

${ }^{2}$ Traits are presented as the percentage of time spent in different behavioural activities

RNN $=$ Rhode Island Red $\times$ Naked Neck; BNN $=$ Black Australorp $\times$ Naked Neck; NN $=$ Naked Neck; FR $=$ Free Range; SI $=$ SemiIntensive; I = Intensive; WAK = Walking; JMP = Jumping; RUN = Running; FD = Feeding; STD = Standing; SIT = Sitting; AGR = Aggressiveness; DB = Dust Bathing; WF $=$ Wing Flapping. 
Table 7. Effect of genotype and housing system on female behavioural traits (7-16 weeks $\left.{ }^{1}\right)$.

\begin{tabular}{|c|c|c|c|c|c|c|c|c|}
\hline \multirow{2}{*}{ Trait $^{2}$} & \multicolumn{3}{|c|}{ Genotype } & \multirow{2}{*}{ P-value } & \multicolumn{3}{|c|}{ Housing System } & \multirow{2}{*}{ P-value } \\
\hline & RNN $(n=60)$ & BNN $(n=60)$ & NN $(n=60)$ & & FR $(n=60)$ & SI $(n=60)$ & $I(n=60)$ & \\
\hline WAK & $13.09 \pm 1.29$ & $13.11 \pm 1.29$ & $13.14 \pm 1.29$ & 0.657 & $14.77 \pm 0.04^{\mathrm{b}}$ & $24.34 \pm 0.05^{\mathrm{a}}$ & $0.23 \pm 0.00^{c}$ & $<0.001$ \\
\hline JMP & $0.89 \pm 0.08$ & $0.93 \pm 0.09$ & $0.91 \pm 0.09$ & 0.770 & $1.25 \pm 0.04^{\mathrm{b}}$ & $1.45 \pm 0.03^{\mathrm{a}}$ & $0.03 \pm 0.00^{\mathrm{c}}$ & $<0.001$ \\
\hline RUN & $6.66 \pm 0.66^{\mathrm{a}}$ & $6.65 \pm 0.65^{\mathrm{a}}$ & $6.52 \pm 0.65^{b}$ & 0.047 & $7.77 \pm 0.07^{\mathrm{b}}$ & $12.06 \pm 0.05^{\mathrm{a}}$ & $0.00 \pm 0.00^{c}$ & $<0.001$ \\
\hline FD & $17.58 \pm 0.54$ & $17.56 \pm 0.54$ & $17.60 \pm 0.54$ & 0.806 & $23.10 \pm 0.05^{\mathrm{a}}$ & $16.47 \pm 0.04^{\mathrm{b}}$ & $13.17 \pm 0.04^{\mathrm{c}}$ & $<0.001$ \\
\hline STD & $12.45 \pm 1.18$ & $12.48 \pm 1.19$ & $12.45 \pm 1.18$ & 0.740 & $6.39 \pm 0.03^{\mathrm{b}}$ & $5.72 \pm 0.02^{\mathrm{c}}$ & $25.30 \pm 0.04^{\mathrm{a}}$ & $<0.001$ \\
\hline SIT & $11.55 \pm 0.90$ & $11.53 \pm 0.90$ & $11.57 \pm 0.91$ & 0.709 & $7.30 \pm 0.03^{\mathrm{b}}$ & $6.02 \pm 0.02^{\mathrm{c}}$ & $21.33 \pm 0.05^{\mathrm{a}}$ & $<0.001$ \\
\hline AGR & $1.53 \pm 0.06$ & $1.54 \pm 0.07$ & $1.51 \pm 0.06$ & 0.496 & $1.04 \pm 0.01^{\mathrm{c}}$ & $1.36 \pm 0.01^{\mathrm{b}}$ & $2.18 \pm 0.02^{\mathrm{a}}$ & $<0.001$ \\
\hline DB & $14.84 \pm 0.25$ & $14.78 \pm 0.23$ & $14.80 \pm 0.24$ & 0.767 & $16.28 \pm 0.05^{\mathrm{a}}$ & $13.34 \pm 0.05^{\mathrm{b}}$ & -- & $<0.001$ \\
\hline WF & $4.33 \pm 0.37$ & $4.29 \pm 0.36$ & $4.29 \pm 0.36$ & 0.571 & $6.53 \pm 0.03^{\mathrm{a}}$ & $5.99 \pm 0.04^{\mathrm{b}}$ & $0.39 \pm 0.00^{\mathrm{c}}$ & $<0.001$ \\
\hline
\end{tabular}

${ }^{\mathrm{a}-\mathrm{c}}$ Means in a row with no common superscript differ significantly at $\mathrm{P} \leq 0.05$.

${ }^{1}$ Values are the least-square mean \pm standard error, the average of each behaviour repertoire from 7-16 weeks.

${ }^{2}$ Traits are presented as the percentage of time spent in different behavioural activities

RNN $=$ Rhode Island Red $\times$ Naked Neck; BNN = Black Australorp $\times$ Naked Neck; NN = Naked Neck; FR = Free Range; SI = Semi Intensive; I = Intensive; WAK = Walking; JMP = Jumping; RUN = Running; FD = Feeding; STD = Standing; SIT = Sitting; AGR = Aggressiveness; DB = Dust Bathing; WF = Wing Flapping.

Table 8. Interaction effects (genotype $\times$ housing system) on female behavioural traits $\left(7-16\right.$ weeks $\left.{ }^{1}\right)$.

\begin{tabular}{|c|c|c|c|c|c|c|c|c|c|c|}
\hline \multirow[b]{2}{*}{ Trait $^{2}$} & \multicolumn{3}{|c|}{ RNN } & \multicolumn{3}{|c|}{ BNN } & \multicolumn{3}{|c|}{$\mathbf{N N}$} & \multirow[b]{2}{*}{ P-value } \\
\hline & $\begin{array}{c}\text { FR } \\
(n=20)\end{array}$ & $\begin{array}{c}\text { SI } \\
(n=20)\end{array}$ & $\begin{array}{c}I \\
(\mathbf{n}=\mathbf{2 0})\end{array}$ & $\begin{array}{c}\text { FR } \\
(n=20)\end{array}$ & $\begin{array}{c}\text { SI } \\
(n=20)\end{array}$ & $\begin{array}{c}I \\
(\mathbf{n}=\mathbf{2 0})\end{array}$ & $\begin{array}{c}\text { FR } \\
(n=20)\end{array}$ & $\begin{array}{c}\text { SI } \\
(\mathbf{n}=\mathbf{2 0})\end{array}$ & $\begin{array}{c}I \\
(n=20)\end{array}$ & \\
\hline WAK & $\begin{array}{c}14.73 \\
\pm 0.08^{\mathrm{b}}\end{array}$ & $\begin{array}{c}24.32 \\
\pm 0.07^{\mathrm{a}}\end{array}$ & $\begin{array}{c}0.22 \\
\pm 0.01^{\mathrm{c}}\end{array}$ & $\begin{array}{c}14.79 \\
\pm 0.08^{b}\end{array}$ & $\begin{array}{c}24.30 \\
\pm 0.09^{\mathrm{a}}\end{array}$ & $\begin{array}{c}0.23 \\
\pm 0.01^{\mathrm{c}}\end{array}$ & $\begin{array}{c}14.79 \\
\pm 0.08^{\mathrm{b}}\end{array}$ & $\begin{array}{c}24.38 \\
\pm 0.08^{\mathrm{a}}\end{array}$ & $\begin{array}{c}0.23 \\
\pm 0.01^{\mathrm{c}}\end{array}$ & $<0.001$ \\
\hline JMP & $\begin{aligned} & 1.27 \\
\pm & 0.07^{\mathrm{ab}}\end{aligned}$ & $\begin{aligned} & 1.38 \\
\pm & 0.05^{\mathrm{ab}}\end{aligned}$ & $\begin{array}{c}0.03 \\
\pm 0.00^{\mathrm{c}}\end{array}$ & $\begin{aligned} & 1.28 \\
\pm & 0.07^{\mathrm{ab}}\end{aligned}$ & $\begin{array}{c}1.46 \\
\pm 0.06^{\mathrm{a}}\end{array}$ & $\begin{array}{c}0.03 \\
\pm 0.00^{\mathrm{c}}\end{array}$ & $\begin{array}{c}1.21 \\
\pm 0.07^{\mathrm{b}}\end{array}$ & $\begin{array}{c}1.51 \\
\pm 0.06^{\mathrm{a}}\end{array}$ & $\begin{array}{c}0.03 \\
\pm 0.06^{\mathrm{c}}\end{array}$ & $<0.001$ \\
\hline RUN & $\begin{array}{c}7.84 \\
\pm 0.10^{\mathrm{b}}\end{array}$ & $\begin{array}{c}12.15 \\
\pm 0.10^{\mathrm{a}}\end{array}$ & $\begin{array}{c}0.00 \\
\pm 0.00^{\mathrm{c}}\end{array}$ & $\begin{array}{c}7.90 \\
\pm 0.12^{\mathrm{b}}\end{array}$ & $\begin{array}{c}12.05 \\
\pm 0.07^{\mathrm{a}}\end{array}$ & $\begin{array}{c}0.00 \\
\pm 0.00^{\mathrm{c}}\end{array}$ & $\begin{array}{c}7.57 \\
\pm 0.11^{\mathrm{b}}\end{array}$ & $\begin{array}{c}11.98 \\
\pm 0.06^{\mathrm{a}}\end{array}$ & $\begin{array}{c}0.00 \\
\pm 0.00^{\mathrm{c}}\end{array}$ & $<0.001$ \\
\hline FD & $\begin{array}{c}23.12 \\
\pm 0.09^{\mathrm{a}}\end{array}$ & $\begin{array}{c}16.48 \\
\pm 0.07^{\mathrm{b}}\end{array}$ & $\begin{array}{c}13.14 \\
\pm 0.05^{\mathrm{c}}\end{array}$ & $\begin{array}{c}23.02 \\
\pm 0.08^{\mathrm{a}}\end{array}$ & $\begin{array}{c}16.51 \\
\pm 0.06^{b}\end{array}$ & $\begin{array}{c}13.14 \\
\pm 0.11^{\mathrm{c}}\end{array}$ & $\begin{array}{c}23.16 \\
\pm 0.09^{\mathrm{a}}\end{array}$ & $\begin{array}{c}16.42 \\
\pm 0.07^{\mathrm{b}}\end{array}$ & $\begin{array}{c}13.22 \\
\pm 0.06^{\mathrm{c}}\end{array}$ & $<0.001$ \\
\hline STD & $\begin{array}{c}6.45 \\
\pm 0.05^{\mathrm{b}}\end{array}$ & $\begin{array}{c}5.70 \\
\pm 0.03^{\mathrm{c}}\end{array}$ & $\begin{array}{c}25.31 \\
\pm 0.07^{\mathrm{a}}\end{array}$ & $\begin{array}{c}6.34 \\
\pm 0.04^{\mathrm{b}}\end{array}$ & $\begin{array}{c}5.73 \\
\pm 0.03^{\mathrm{c}}\end{array}$ & $\begin{array}{c}25.36 \\
\pm 0.08^{\mathrm{a}}\end{array}$ & $\begin{array}{c}6.40 \\
\pm 0.05^{\mathrm{b}}\end{array}$ & $\begin{array}{c}5.72 \\
\pm 0.03^{\mathrm{c}}\end{array}$ & $\begin{array}{c}25.24 \\
\pm 0.08^{\mathrm{a}}\end{array}$ & $<0.001$ \\
\hline SIT & $\begin{array}{c}7.30 \\
\pm 0.06^{\mathrm{b}}\end{array}$ & $\begin{array}{c}6.02 \\
\pm 0.03^{\mathrm{c}}\end{array}$ & $\begin{array}{l}21.35 \\
\pm 0.09^{\mathrm{a}}\end{array}$ & $\begin{array}{c}7.30 \\
\pm 0.05^{\mathrm{b}}\end{array}$ & $\begin{array}{c}6.04 \\
\pm 0.02^{\mathrm{c}}\end{array}$ & $\begin{array}{c}21.25 \\
\pm 0.08^{\mathrm{a}}\end{array}$ & $\begin{array}{c}7.31 \\
\pm 0.04^{\mathrm{b}}\end{array}$ & $\begin{array}{c}6.02 \\
\pm 0.03^{\mathrm{c}}\end{array}$ & $\begin{array}{c}21.38 \\
\pm 0.08^{\mathrm{a}}\end{array}$ & $<0.001$ \\
\hline AGR & $\begin{array}{c}1.03 \\
\pm 0.02^{\mathrm{c}}\end{array}$ & $\begin{array}{c}1.38 \\
\pm 0.01^{\mathrm{b}}\end{array}$ & $\begin{array}{c}2.18 \\
\pm 0.04^{\mathrm{a}}\end{array}$ & $\begin{array}{c}1.05 \\
\pm 0.02^{\mathrm{c}}\end{array}$ & $\begin{array}{c}1.36 \\
\pm 0.02^{\mathrm{b}}\end{array}$ & $\begin{array}{c}2.21 \\
\pm 0.04^{\mathrm{a}}\end{array}$ & $\begin{array}{c}1.06 \\
\pm 0.01^{\mathrm{c}}\end{array}$ & $\begin{array}{c}1.34 \\
\pm 0.02^{\mathrm{b}}\end{array}$ & $\begin{array}{c}2.14 \\
\pm 0.03^{\mathrm{a}}\end{array}$ & $<0.001$ \\
\hline DB & $\begin{array}{c}16.40 \\
\pm 0.08^{\mathrm{a}}\end{array}$ & $\begin{array}{c}13.29 \\
\pm 0.07^{\mathrm{b}}\end{array}$ & -- & $\begin{array}{c}16.17 \\
\pm 0.09^{\mathrm{a}}\end{array}$ & $\begin{array}{c}13.40 \\
\pm 0.10^{\mathrm{b}}\end{array}$ & -- & $\begin{array}{c}16.28 \\
\pm 0.08^{\mathrm{a}}\end{array}$ & $\begin{array}{c}13.32 \\
\pm 0.09^{\mathrm{b}}\end{array}$ & -- & $<0.001$ \\
\hline WF & $\begin{array}{c}6.58 \\
\pm 0.06^{\mathrm{a}}\end{array}$ & $\begin{array}{c}6.03 \\
\pm 0.08^{\mathrm{b}}\end{array}$ & $\begin{array}{c}0.39 \\
\pm 0.00^{\mathrm{c}}\end{array}$ & $\begin{array}{c}6.52 \\
\pm 0.05^{\mathrm{a}}\end{array}$ & $\begin{array}{c}5.98 \\
\pm 0.07^{\mathrm{b}}\end{array}$ & $\begin{array}{c}0.39 \\
\pm 0.00^{\mathrm{c}}\end{array}$ & $\begin{array}{c}6.50 \\
\pm 0.05^{\mathrm{a}}\end{array}$ & $\begin{aligned} & 5.98 \\
\pm & 0.08^{\mathrm{b}}\end{aligned}$ & $\begin{array}{c}0.39 \\
\pm 0.00^{\mathrm{c}}\end{array}$ & $<0.001$ \\
\hline
\end{tabular}

${ }_{\mathrm{a}-\mathrm{c}}$ Means in a row with no common superscript differ significantly at $\mathrm{P} \leq 0.05$.

${ }^{1}$ Values are the least-square mean \pm standard error, the average of each behaviour repertoire from 7-16 weeks.

${ }^{2}$ Traits are presented as the percentage of time spent in different behavioural activities

$\mathrm{RNN}=$ Rhode Island Red $\times$ Naked Neck; BNN = Black Australorp $\times$ Naked Neck; NN = Naked Neck; FR = Free Range; SI = Semi Intensive; I = Intensive; WAK = Walking; JMP = Jumping; RUN = Running; FD = Feeding; STD = Standing; SIT = Sitting; AGR = Aggressiveness; DB = Dust Bathing; WF $=$ Wing Flapping.

Sitting $(\mathrm{P}<0.001)$ and standing $(\mathrm{P}<0.001)$ behaviours were more pronounced in the intensive system birds followed by free-range and semi-intensive systems. Birds under semi-intensive spent most of their time in walking $(\mathrm{P}<0.001)$ and wing-flapping behaviour following by freerange and intensive systems. Interactions were significant $(\mathrm{P}<0.001)$ between genotypes and housing systems regarding walking, jumping, running, feeding, standing, sitting, aggressiveness, dust bathing, and wing flapping.

Regarding females, a significant difference was observed regarding running behaviour among different genotypes. RNN and BNN chicken different and revealed more intense running behaviour than $\mathrm{NN}(6.66,6.65$ vs. $6.52 \% ; \mathrm{P}=0.047)$. In terms of housing systems, significant 
differences were observed regarding aggressiveness, dust bathing, feeding, jumping, running, sitting, standing, walking, and wing flapping. Birds reared under the intensive systems were more aggressive followed by semiintensive and free-range systems $(\mathrm{P}<0.001)$. Dust bathing was more pronounced in free-range birds than the semiintensive systems (16.28 vs. $13.34 \%$; $<<0.001)$. Birds under the free-range system spent most of their time feeding $(\mathrm{P}<0.001)$ and wing flapping $(\mathrm{P}<0.001)$ followed by semi-intensive and intensive housing systems. Jumping ( $\mathrm{P}<0.001)$, running $(\mathrm{P}<0.001)$, and walking $(\mathrm{P}<0.001)$ were more pronounced in the semi-intensive system followed by a free-range and intensive system. Sitting $(\mathrm{P}<0.001)$ and standing $(\mathrm{P}<0.001)$ behaviour were higher in birds under intensive system followed by free-range and semi-intensive systems. Interactions were significant $(\mathrm{P}<0.001)$ between genotypes and housing systems regarding walking, jumping, running, feeding, standing, sitting, aggressiveness, dust bathing, and wing flapping.

\section{Discussion and Conclusion}

The present study aimed to explore the genetic potential of different chicken genotypes under alternative production systems and this was successful as the behaviour of different genotypes was astonishing when subjected to different housing systems. Free-range birds spent more time in dust bathing than semi-intensive chickens and this could be due to infrequent behaviour which is initiated when birds given access to the ample space for movement and birds have the opportunity to find material for its cleaning such as dust. Moreover, the reason for this result may be the length of time the birds were spending outside the facility. Bathing is usually done in the afternoon, and the birds from the semi-intensive system were not outside at that time. According to RSPCA (17) domestic chicken has intrinsic motivation for cleaning its feathers. This corresponds to the findings of Appleby et al. (2) who reported that an intensive system is not suitable for the birds because it restricts the expression of natural behaviours like dust bathing.

Feeding behaviour is more pronounced in free-range birds followed by a semi-intensive and intensive system. It might be due to the variety of stimuli in the range area which provokes foraging behaviour in the birds. Furthermore, due to ample space in the free-range system, it provides lots of opportunities for the birds to initiate their explorative behaviour. Similar findings were also suggested that foraging behaviour increased in the commercial broiler with the provision of the free-range area as compared to confinement (14). Moreover, Shimmura et al. (20) supported to enhance the feeding behaviour of commercial layers when given access to a free-range system.
Jumping, running walking, and wing-flapping behaviour was more pronounced in semi-intensive birds followed by the free-range and intensive system. The more likely explanation of these behaviours is because when birds are provided with an enriched environment or outdoor access it promotes comfort level, reduces stress, and stimulating activities. This corresponds to the findings of Irfan et al. (9) who found increased immobility in turkey (Meleagris gallopavo) maintained in confinement than free-range birds. Moreover, Mench et al. (11) reported that the frequency of leg stretching, perching, and wing flapping of broiler chicken increased when given access to an outdoor range. Similarly, an enhanced walking behaviour of Aseel chicken was recorded when subjected to part-time free-range access (16).

In this study, increased aggressive, sitting, and standing behaviour in birds with an intensive housing system might be attributed to the higher stocking density that restricts the bird's activity and stimulates short duration behaviour such as aggression. This corresponds to the findings of Rehman et al. (16) who found enhance sitting and standing behaviour of Aseel chicken varieties reared under confinement. On an overall basis, the behavioural repertoire did not differ among RNN, BNN, and NN genotype throughout the experimental period. However, another study (16) reported a higher frequency of aggressive behaviour in crossbred chicken (cross between Thai native male "Luang Hang Khao or Whitetailed Yellow breed" and ISA Brown Commercial layer type female).

It was concluded that $\mathrm{RNN}$ and $\mathrm{BNN}$ chickens expressed more natural behaviours under semi-intensive and free-range systems than NN chickens; hence, crossbred chickens could be reared under such types of environment to achieve their maximum genetic potential.

\section{Acknowledgments}

This study is a part of the Doctoral Thesis of the first author. The authors gratefully acknowledge the administration at Indigenous Chicken Genetic Resource Centre, the University of Veterinary and Animal Sciences, Ravi Campus, Pattoki for their facilitation during this trial.

\section{Financial Support}

This study was financially supported by Pakistan Agricultural Research Council, ALP Fund (Project No. AS-135).

\section{Ethical Statement}

The care and use of birds were by the laws and regulations of Pakistan and 101 was approved by the Committee of Ethical Handling of Experimental Birds 
(No. 102 DR/124), the University of Veterinary and Animal Sciences (UVAS), Lahore, Pakistan.

\section{Conflict of Interest}

The authors declared that there is no conflict of interest.

\section{References}

1. Anonymous (2011): South African show poultry organisation breed standards. Available at: http://www.saspo.org.za. (Accessed September 06, 2020).

2. Appleby MC, Mench JA, Hughes BO (2004): Poultry Behaviour and Welfare. 118-175. In: Perceptions of Welfare. CABI Publishing, Oxfordshire, UK.

3. Bogosavljević-Bošković S, Rakonjac S, Dosković V, et al (2012): Broiler rearing systems: A review of major fattening results and meat quality traits. Worlds Poult Sci J, 68, 217228.

4. Costa LS, Pereira DF, Bueno LGF, et al (2012): Some aspects of chicken behaviour and welfare. Braz J Poult Sci, 14, 159-164.

5. Dohner JV (2010): Black Australorp chickens: Heritage poultry breeds. Available at: https://www.motherearthnews.com/homesteading-andlivestock/raising-chickens/black-australorp-heritagepoultry-zeylaf. (Accessed September 06, 2020).

6. El-Deek A, El-Sabrout K (2019): Behaviour and meat quality of chicken under different housing system. Worlds Poult Sci J, 75, 1-9.

7. Fanatico AC, Pillai PB, Cavitt LC, et al (2006): Evaluation of slower growing broiler genotypes grown with and without outdoor access: sensory attributes. Poult Sci, 85, 337-343.

8. Huo X, Na-Lampang P (2016): Effects of stocking density on feather pecking and aggressive behaviour in Thai crossbred chickens. Agric Nat Res, 50, 396-399.

9. Javid A, Ashraf M, Mahmud, A, et al (2016): Timebudgets of turkeys (Maleagris gallopavo) reared under confinement and free range rearing systems. Pak J Zool, 48, 1951-1956.

10. Leeson S, Summers JD (2005): Commercial Poultry Nutrition. $3^{\text {rd }}$ Edition. Nottingham University Press, Nottingham, England.

11. Mench JA, Garner JP, Falcone C (2001): Behavioural activity and its effects on leg problems in broiler chickens.
152-156. In: Proceedings of $6^{\text {th }}$ European Symposium Poultry Welfare Zollikofen, Switzerland.

12. Mohammed AA, Jacobs JA, Murugesan GR, et al (2018): Effect of dietary synbiotic supplement on behavioural patterns and growth performance of broiler chickens reared under heat stress. Poult Sci, 97, 1101-1108.

13. Momoh OM, Nwosu CC (2008): Genetic evaluation of growth traits in crosses between two ecotypes of Nigerian local chicken. Livestock Res Rural Dev, 20, 580-690.

14. Ponte PIP, Rosado CMC, Crespo JP, et al (2008): Pasture intake improves the performance and meat sensory attributes of free-range broilers. Poult Sci, 87, 71-79.

15. Razuki WM, Mukhlis SA, Jasmin FH, et al (2011): Productive performance of four commercial broiler genotypes reared under high ambient temperatures. Int $\mathbf{J}$ Poult Sci, 10, 87-92.

16. Rehman MS, Mahmud A, Mehmood S, et al (2018): Assessing behaviour in Aseel pullets under free-range, parttime free-range, and cage system during growing phase. Poult Sci, 97, 725-732.

17. RSPCA (2016): The Welfare of Layer Hens in Cage and Cage-Free Housing Systems. RSPCA Australia, Deakin West ACT 2600, Australia.

18. Saadey SM, Galal A, Zaky HI, et al (2008): Diallel crossing analysis for body weights and egg production traits of two native Egyptian and two exotic chicken breeds. Int J Poult Sci, 7, 64-71.

19. Shafiq M, Mahmud A, Hussain J, et al (2018): Comparative production performance of four different Naked Neck chicken phenotypes in Pakistan. J Anim Plant Sci, 28, 33-37.

20. Shimmura T, Suzuki T, Hirahara S, et al (2008): Pecking behaviour of laying hens in single-tiered aviaries with and without outdoor area. Brit Poult Sci, 49, 396-401.

21. Siwendu NA, Norris D, Ng'Ambi JW, et al (2012): Heterotic and combining ability for body weight in a diallel cross of three chicken genotypes. Trop Anim Health Prod, 20, 23-25.

22. Tukey JW (1953): The problem of multiple comparisons. The Collected Works of JOHN W. Tukey VIII. Multiple Comparisons. Chapman and Hall, New York.

23. Van Marle-Köster E, Hefer CA, Nel LH, et al (2008): Genetic diversity and population structure of locally adapted South African chicken lines: Implications for conservation. South Afr J Anim Sci, 38, 271-281. 